This item was submitted to Loughborough's Research Repository by the author.

Items in Figshare are protected by copyright, with all rights reserved, unless otherwise indicated.

\title{
Maintenance modelling of complex automated guided vehicle systems
}

PLEASE CITE THE PUBLISHED VERSION

https://doi.org/10.1109/RAMS.2019.8769020

PUBLISHER

IEEE

VERSION

AM (Accepted Manuscript)

PUBLISHER STATEMENT

(c) 2019 IEEE. Personal use of this material is permitted. Permission from IEEE must be obtained for all other uses, in any current or future media, including reprinting/republishing this material for advertising or promotional purposes, creating new collective works, for resale or redistribution to servers or lists, or reuse of any copyrighted component of this work in other works.

\section{LICENCE}

All Rights Reserved

REPOSITORY RECORD

Yan, Rundong (Derek), Sarah Dunnett, and Lisa Jackson. 2019. "Maintenance Modelling of Complex Automated Guided Vehicle Systems". figshare. https://hdl.handle.net/2134/37032. 


\section{Maintenance modelling of complex automated guided vehicle systems}

\author{
Rundong Yan, Loughborough University \\ Sarah Dunnett, Loughborough University \\ Lisa Jackson, Loughborough University
}

Key Words: Automated guided vehicles, maintenance, petri nets

\section{SUMMARY \& CONCLUSIONS}

Automated guided vehicles (AGV's) have been adopted in many industrial applications since their introduction in the 1950's. Although still primarily used for the movement of materials around manufacturing facilities and warehouses they are also used in such applications as hospitals and transportation. Such driverless vehicles generally travel along a predefined route performing set tasks and they have been widely adopted due to their efficiency and economic benefits, Le-Anh and De Koster (2006). The availability of the vehicles is crucial to ensure that these benefits are maintained. As the complexity of industrial processes increases and fleets of AGV's are commonly employed, maintenance and reliability issues are of increasing concern. In order to ensure that the benefits of AGV's are utilised efficiently it is crucial that efficient maintenance strategies are employed. Hence in this work research has been undertaken into determining the optimal maintenance strategy for a complex multi AGV system.

Typically a multi AGV system will consist of a number of vehicles that travel along the same route performing required tasks. Once any AGV fails it should be removed from the route as quickly as possible in order to prevent obstructing other AGV's. In this work Coloured Petri Nets (CPN) and Genetic Algorithms are used in combination in order to determine the optimal maintenance strategy. From the research conducted it is found that the maintenance strategies adopted and the location of the maintenance site are significant factors impacting on the efficiency, cost, and productivity of a multi-AGV system.

\section{INTRODUCTION}

Automated guided vehicles are widely used in many industries, in particular in material handling, due to their flexibility and efficiency. With the increasing application of AGV systems, and their subsequent increase in fleet size, reliability and maintenance issues are of increasing importance. Past work on AGV systems has concentrated on facility layout design in terms of travel time [1-3] with reliability and maintenance receiving less attention. Relevant work in this area includes [4] where the reliability of AGV's was modelled as a cost function in order to optimize their operation, and [5] where a control method was developed for enhancing the failure control management of underground transport AGV's. Due to the lack of understanding of the reliability issues affecting AGV's the authors adopted a combined fault tree and petri net approach to analyse system and mission reliability in [6]. A typical AGV transport system was considered which consisted of eight subsystems; laser navigation, safety system, software control system, drive unit, brake system, steering system, attachments and batteries. Fault trees were constructed describing the failure of each subsystem and these were then used to build a petri net model to determine mission reliability for an AGV. Although this initial study only considered a single AGV the methods adopted were found to be efficient and flexible and further work [7] extended the model to consider multi AGV systems. In the work presented here the earlier work is built upon and the optimal maintenance strategy is investigated for a multi AGV system. A simulation model has been used to obtain results for various scenarios that are then fed into a genetic algorithm in order to optimize the maintenance strategy.

\section{AGV SYSTEM AND MISSION}

A multi AGV system is considered consisting of $\mathrm{N}_{\mathrm{a}}$ vehicles. The vehicles are assumed to have the same structure as those considered in [6] and hence the model described in that work could be used to determine their failure rate. In this work the failure rate is taken to be 8 per year. The probability of failure is then assumed to follow a Weibull distribution. Considering a multi AGV system means that interactions between vehicles must be considered in particular the effect one AGV failure has upon others. In the model developed here it is assumed that once an AGV fails it is removed from the route as soon as possible by a 'recycle vehicle' in order to minimize the downtime of the system. In order to achieve this, the location of the maintenance site, where the 'recycle vehicle' is housed and the failed vehicle taken to, is crucial. In this work three different configurations are considered. Typically an AGV travels from its base to a station, 'pickup station' where it collects material, it then takes the material to its destination, 'storage site', where it unloads and then returns to its base. Hence the three configurations considered all consist of the locations; base, pickup station, storage site and maintenance site and the paths between these sites. These are shown in Figure 1 where MS indicates the location of the 
maintenance site. The different layouts are distinguished by the location of MS and the extra paths for recycling failed AGV's.

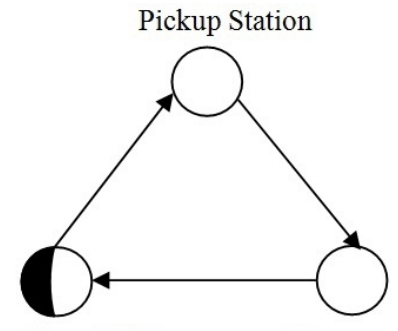

Base \& MS

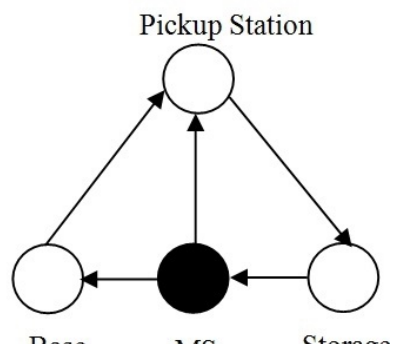

(b) Configuration 2 (a) Configuration 1

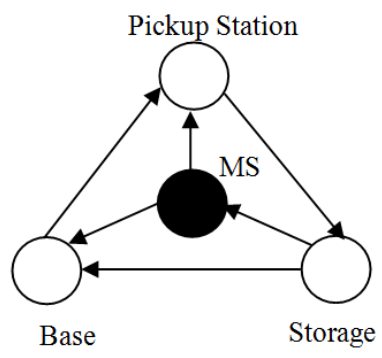

(c) Configuration 3

Figure 1. Layout configurations considered

As in [6] the mission undertaken by the AGV's is assumed to comprise of six phases, as shown in table 1 where the length of each phase is also given. The times given in Table 1 have been assumed in order to run the model developed and will be different depending upon facility.

\begin{tabular}{lll}
\hline Phase & Task & Phase Length (hours) \\
\hline 1 & $\begin{array}{l}\text { Mission allocation and } \\
\text { route optimisation }\end{array}$ & 0.04 \\
2 & Dispatch to station & 0.4 \\
3 & Loading of item & 0.04 \\
4 & Travelling to storage & 0.4 \\
5 & Unloading & 0.04 \\
6 & Return to base & 0.4 \\
\hline
\end{tabular}

Table 1.Phase definition and length

\section{SYSTEM MODEL}

Petri net modelling has been identified in previous work to be an efficient method to model AGV systems [6-8]. Such models are dynamic and highly adaptive and provide an intuitive graphical representation of a system. For complex systems or systems designed to carry out complex tasks or missions Coloured Petri nets (CPN) have been shown to be efficient [9] and hence have been adopted here. Such models are made up of: places, which may be conditions or states, represented by circles; transitions, actions which cause a change of condition or state, represented by rectangles; arcs connecting places and transitions, represented by arrows and tokens, which carry the information in the net, represented by small circles. If the time for completing the transition is instant the rectangle is filled in otherwise it is hollow. The tokens move through the net and the marking of the net at any particular time gives the state of the system being modelled. The movement of the tokens is governed by the enabling of transitions. A transition is said to be enabled if the places that input to the transition all contain a number of tokens equal or greater than the corresponding arc weight of the arc connecting the place and the transition. The weight of an arc is the number of arcs that connect a place and a transition and are generally represented on a net by a slash through the arc with the weight number next to it. If an arc has no slash on it then the weight of the arc is one. Once a transition is enabled then after any delay associated with that transition it will fire and take tokens from the input places equivalent to their arc weights and place tokens in the output places equivalent to the output arc weights. An example of this movement of tokens is shown in Figure 2.

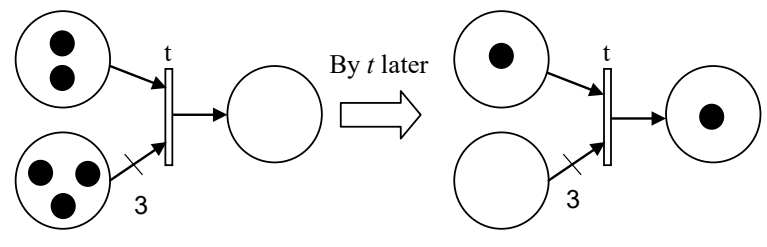

Figure 2. Example of an enabled transition.

In CPN's the tokens are assigned colours which carry specific information. A CPN model has been developed to describe the operation and maintenance activities in a multi AGV system by the authors in [7]. This is briefly outlined below. The model consists of five linked nets:

1. Path Petri Nets (PPN) - these describe the configuration of the system.

2. Master Petri Net (MPN) - this governs the mission progress or phase change of individual AGV's

3. Recycle Petri Net (RPN) - this describes the recycling process of failed AGV's.

4. Corrective maintenance Petri Net (CMPN) describes the corrective maintenance process

5. Periodic maintenance Petri Net (PMPN) - describes the periodic maintenance process

\subsection{Path Petri Nets (PPN)}

PPN's have been constructed to model the three layout configurations shown in Figure 1. As an example Figure 3 shows the net for the configuration shown in Figure 1b. The dotted arrows in the figure represent the flow of information to and from the other nets. In the net shown there are 3 tokens of different colours representing 3 different AGV's. These start at the base as shown, when a token moves into the place representing phase 2 in the MPN then the transition between the places 'base' and 'pickup station' is enabled and a token of the same colour will move between the places. Hence modelling the movement of the AGV's around the facility. The other transitions operate in the same manner. 


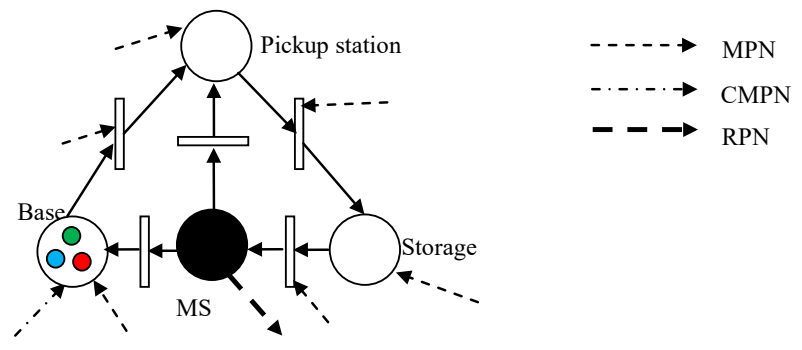

Figure 3. PPN for the layout configuration in Figure $1 b$

\subsection{Master Petri Net (MPN)}

As detailed in Table 1 the mission of the AGV's is comprised of 6 phases, the MPN models the progression of the mission through the phases to either mission complete or mission failure. Figure 4 shows the MPN, in the figure its link with the PPN from figure 3 is also included in order to demonstrate the integration. In the figure the 'base' place which is shown twice in the figure is the same place but has been repeated here in order to try to clarify the figure and reduce the overlapping arrows.

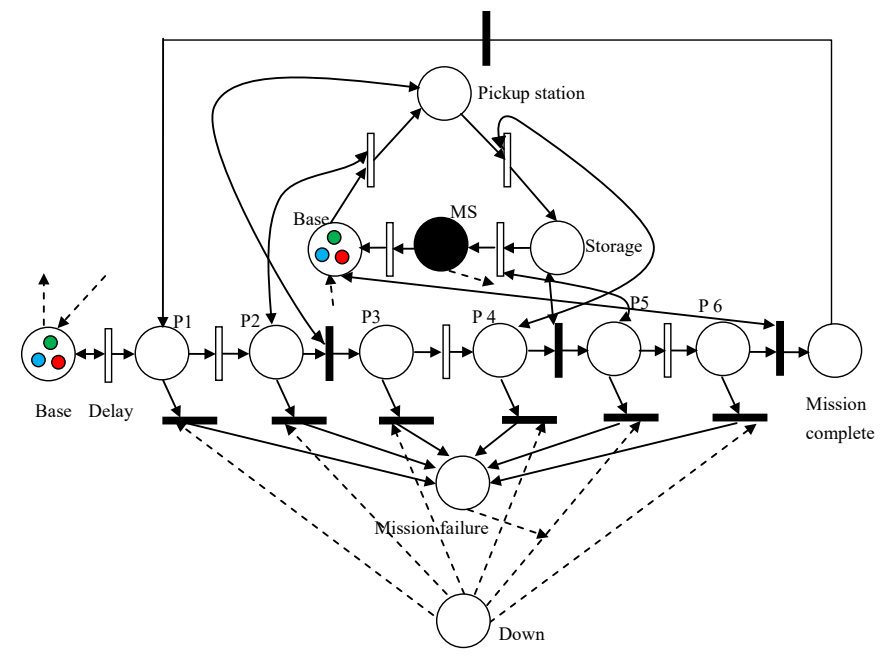

Figure 4. MPN combined with PPN

The tokens inside the 'base' place indicate that there are AGV's free to be allocated to a mission. Only the same coloured tokens in the MPN and the PPN can enable transitions enabling the movement of AGV's and their working phases to be correlated. For example, once a token is placed into the 'P2' place, representing dispatch to station, then the transition between the 'base' and 'pickup station' places in the PPN is enabled. After the delay associated with travelling between the base and the station has expired this transition will fire and the token will be removed from ' $\mathrm{P} 2$ ' and a token of the same colour will be removed from 'base'. Tokens of the same colour will then be placed in 'P2' and 'pickup station'. The progression through the mission carries on in the same way. Once an AGV fails the corresponding token for that vehicle will reside in the place 'down' hence enabling a transition to 'mission failure' for the appropriate phase. The 'mission failure' place is connected to the RPN, as indicated by the dotted arrow from the place, ensuring that once a token moves into this place the recycling process of the failed AGV is started.

\subsection{Recycle Petri Net (RPN)}

This net describes the process undertaken once an AGV fails and needs to be removed to the maintenance site. For space reasons the net has not been included here but is described briefly below, more detail can be found in [7]. Once an AGV fails and a token resides in the 'mission failed' place in the MPN the recycling of the AGV starts immediately. Initially the position of the failed vehicle is located and the route for the recycle vehicle to take from the maintenance site to the failed vehicle is optimized. If any AGV is found running on the route the recycle vehicle will not leave the maintenance site until that $\mathrm{AGV}$ reaches its next station where it will then park and be removed from the route. Any other vehicles will stop in order to avoid possible blockage. After the recycle vehicle reaches the position of the failed AGV it will tow it back to the maintenance site. As the site can be reached by following the flow of working AGV's all other vehicles restart their mission once the recycle vehicle has collected the failed AGV. As the recycling of failed AGV's disturbs the normal operation of other AGV's the optimal routing of the recycling vehicle is crucial to the performance of the multi-AGV system. In this work the optimal path has been obtained using a forward-tracking search algorithm which is described in [7].

\subsection{Corrective Maintenance Petri Net (CMPN)}

Corrective maintenance will take place for the failed AGV's as soon as they arrive at the maintenance site if an engineer is available, otherwise they will join a queue. On completion of the maintenance the recovered AGV is assumed as good as new. The CMPN developed is shown in Figure 5.

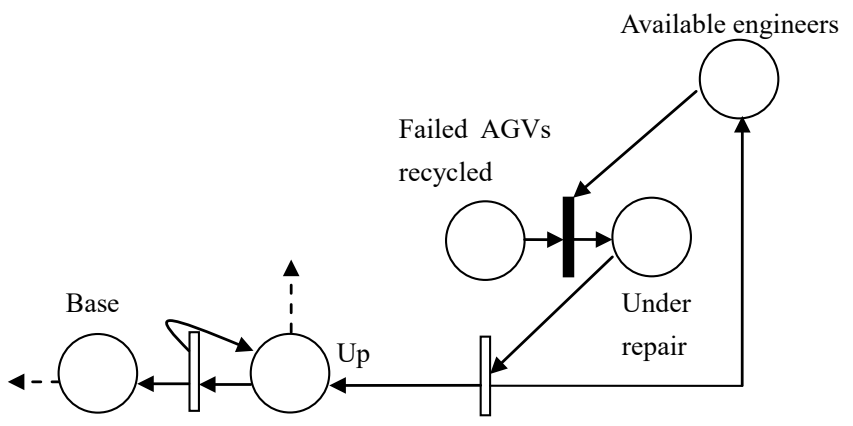

Figure 5. CMPN model

Once a token is placed in the 'failed AGV's recycled' place from the RPN and there is at least one token in the 'available engineers' place then these tokens will be removed and a token placed in the 'under repair' place. Once the time associated with the repair, in this work it is assumed to follow a normal distribution, has elapsed the token in the 'under repair' place is removed and a token put in the places 'up' and 'available engineers'. The token in 'up' place indicates the 
healthy state of the AGV and after a time associated with the travel time back to the base, this token is removed and one placed in the 'base' place indicating that the AGV is ready to perform a mission and one back in the 'up' place.

\subsection{Periodic Maintenance Petri Net (PMPN)}

A PMPN has been developed and after the maintenance has been performed all AGV's are assumed to be as a good as new. All AGV's in the system are assumed to undergo the periodic maintenance whatever their condition. In this work it has been assumed that the maintenance lasts 2 days and various maintenance intervals are considered. The net modelling this process can be seen in [7].

\section{SIMULATION}

By integrating the CPN's described above a model has been developed that can be used to investigate the effect of various parameters, in particular maintenance strategies, upon the performance of multi-AGV systems. An algorithm has been developed to simulate the integrated model which takes as inputs; the failure and repair rates of the AGV's, phase lengths and the time interval between periodic maintenance. An example of the results obtained by the model for the configuration shown in Figure $1 \mathrm{~b}$ for various maintenance regimes for a system of 3 AGV's are given in Table 2.

\begin{tabular}{lllll}
\hline $\mathrm{T}$ & $\mathrm{P}$ & $\mathrm{N} 1$ & $\mathrm{~F}$ & $\mathrm{~N} 2$ \\
\hline 7 days & 0.00 & 6488 & 0.00 & 6570 \\
20 days & 0.00 & 7498 & 0.06 & 7556 \\
1 month & 0.02 & 7656 & 0.20 & 7781 \\
2 months & 8.37 & 7018 & 1.35 & 7962 \\
3 months & 61.88 & 5649 & 2.90 & 8014 \\
4 months & 97.13 & 4373 & 4.23 & 8040 \\
6 months & 100 & 2935 & 7.00 & 8073
\end{tabular}

Table 2. Simulation results for layout in Figure $1 b$

In the table $\mathrm{T}$ is the time interval between periodic maintenance, $\mathrm{P}$ is the percentage of AGV's failed within the time interval if there is no maintenance, N1 is the number of missions completed per year with periodic, but without corrective, maintenance, F1 is the number of failures within the time interval if there is corrective maintenance and N2 is the number of missions completed per year with periodic, and corrective, maintenance. It has been assumed that the system operates for 10 hours per day. From the table it can be seen, for the failure rate assumed, that if no maintenance strategy is applied, within 6 months $100 \%$ of AGV's will fail. This highlights the need for appropriate maintenance to be applied. Also it can be seen by comparing the values of N1 and N2 that corrective maintenance can improve the performance of the multi-AGV system, though this would come at a financial cost.

Investigating the different configuration layouts shown in Figure 1 it was found that time taken to recycle failed AGV's was the shortest for configuration shown in Figure 1c where the maintenance site is placed in the centre. However this configuration involves more routes and the subsequent cost.

The results obtained from the CPN simulations are used to optimize the maintenance strategy.

\section{MAINTENANCE OPTIMISATION}

The aim of the maintenance strategy is to have an efficient and cost effective multi-AGV system. This results in a multi-objective optimization problem. In this work, Genetic Algorithms (GA) have been employed to carry out the optimization. These algorithms have become increasingly popular and have been applied to a wide range of complex real world problems. They use the theories of evolution and natural selection to solve the complex problems. GA have been applied to solving scheduling and dispatching problems for AGV systems. For example, they were applied in [9] to consider simultaneously machine and vehicle scheduling.

The basic principles of GA's were introduced by [10]. To implement the GA optimisation, an initial population of chromosomes is generated. The fitness of each chromosome is evaluated subject to predefined objective functions. By selecting pairs of parents in the population, new chromosomes or children are generated. This is known as crossover. The chromosomes with the higher fitness are more likely to be selected so that their genes can be passed on with higher probability. A mutation might also be involved to prevent early convergence of the solution. Through the process, the chromosomes with larger fitness values are obtained until an optimal solution is reached.

For the multi-AGV system the following two objective functions are defined to optimise the system design in terms of maximising the number of missions completed and minimising the cost.

Objective function 1: Maximise the number of missions completed within a given time

$$
\text { Mission }=\max \left(\mathrm{N}_{\mathrm{m}} \cdot \mathrm{N}_{\mathrm{p}}-\mathrm{T}_{\mathrm{rc}} \cdot \mathrm{N}_{\mathrm{f}} \cdot \mathrm{N}_{\mathrm{a}} / \mathrm{T}_{\mathrm{m}}\right)
$$

Objective function 2: The minimum cost for completing the missions

$$
\begin{gathered}
\mathrm{C}=\mathrm{N}_{\mathrm{p}} \cdot \mathrm{C}_{\mathrm{p}}+\mathrm{N}_{\mathrm{f}} \cdot \mathrm{C}_{\mathrm{C}}+\mathrm{N}_{\mathrm{e}} \cdot \mathrm{C}_{\mathrm{e}}+\mathrm{C}_{\mathrm{ms}}+\mathrm{N}_{\mathrm{m}} \cdot \mathrm{C}_{\mathrm{a}}+\mathrm{L}_{\mathrm{r}} \cdot \mathrm{C}_{\mathrm{r}}+\mathrm{C}_{\mathrm{lm}} \\
\operatorname{Cost}=\min (\mathrm{C})
\end{gathered}
$$

Where $\mathrm{N}_{\mathrm{p}}=365 / \mathrm{T}$ is the number of times periodic maintenance takes place in a year. The other parameters in equations (2) and (3) are given in Table 3 with the values taken in this work.

\begin{tabular}{l|c|l}
\hline Parameters & Symbol & Value \\
\hline Number of AGVs & $N_{a}$ & 3 \\
\hline $\begin{array}{l}\text { Operation cost of an } \\
\text { AGV to complete a } \\
\text { single mission ( })\end{array}$ & $C_{a}$ & 16 \\
\hline $\begin{array}{l}\text { Business costs of } \\
\text { maintenance site per } \\
\text { year (£) }\end{array}$ & $C_{m s}$ & $\begin{array}{l}10000 \text { - with corrective } \\
\text { maintenance } \\
5000 \text { - without corrective } \\
\text { maintenance }\end{array}$ \\
\hline $\begin{array}{l}\text { Land cost for } \\
\text { maintenance site per } \\
\text { year (£) }\end{array}$ & $C_{l m}$ & $\begin{array}{l}1000 \text { - Share site with AGV } \\
\text { base } \\
5000 \text { - Separate site }\end{array}$ \\
\hline \begin{tabular}{l} 
Number of missions \\
\hline
\end{tabular} & $N_{m}$ & Values determined by CPN \\
\hline
\end{tabular}




\begin{tabular}{l|c|l}
\hline competed per year & \multicolumn{2}{|l}{ model. } \\
\hline $\begin{array}{l}\text { Time interval of periodic } \\
\text { maintenance }\end{array}$ & $T$ & $\begin{array}{l}\text { See the values of } \\
\text { T in Table 2 }\end{array}$ \\
\hline Periodic maintenance cost $(£)$ & $C_{p}$ & 2000 \\
\hline Recycle time & $T_{r c}$ & $\begin{array}{l}\text { Values } \\
\text { determined by } \\
\text { CPN model }\end{array}$ \\
\hline $\begin{array}{l}\text { Average time to complete a } \\
\text { mission }\end{array}$ & $T_{m}$ & 1.32 \\
\hline $\begin{array}{l}\text { No. of maintenance engineers } \\
\text { on site }\end{array}$ & $N_{e}$ & 1 \\
\hline $\begin{array}{l}\text { Cost of one Engineer in a year } \\
\text { (£) }\end{array}$ & $C_{e}$ & 25000 \\
\hline $\begin{array}{l}\text { Total number of failures } \\
\text { occurring in the system with } \\
\text { corrective maintenance per year }\end{array}$ & $N_{f}$ & $\begin{array}{l}\text { Values } \\
\text { determined by } \\
\text { Average cost for conducting } \\
\text { corrective maintenance of an } \\
\text { AGV failure (£) }\end{array}$ \\
\hline $\begin{array}{l}\text { Extra route length } \\
\text { CPN model. }\end{array}$ \\
$\begin{array}{l}\text { Cost of per unit length extra } \\
\text { route (£) }\end{array}$ & $C_{c}$ & 800 \\
\hline
\end{tabular}

Table 3. Parameters used in GA program

A fitness function is obtained based on the two objective functions:

$$
\text { fitness }=\text { Mission } / \text { Cost }
$$

The maintenance strategy is optimized subject to the following constraints:

Number of missions per year $\geq 600$

Probability of all AGV's failing $\leq 0.1$

For the three major factors considered, periodic maintenance interval, system configurations and the adoption of corrective maintenance, their values are obtained from the CPN simulations. These three parameters are coded into binary numbers and then connected together to create a single chromosome. The crossover operation is applied to two randomly selected chromosomes with the crossover rate of 0.7. A mutation rate of 0.02 is applied to the mutation operation.

\subsection{Results}

A GA program has been developed optimizing the maintenance strategies of the multi-AGV system using the fitness function integrating the two objective functions, shown in equation (3). In order to demonstrate the effectiveness of the GA optimization an example of some results obtained is given here. Applying the parameters given in Table 3 the population starts to evolve gradually. The average fitness against evolution time is shown in Figure 6.

From Figure 6 it can be seen that the average fitness reaches a saturated value after the population has evolved around 47 times. Hence we can say that the optimal maintenance strategy for the multi-AGV system is obtained after 47 evolution calculations.

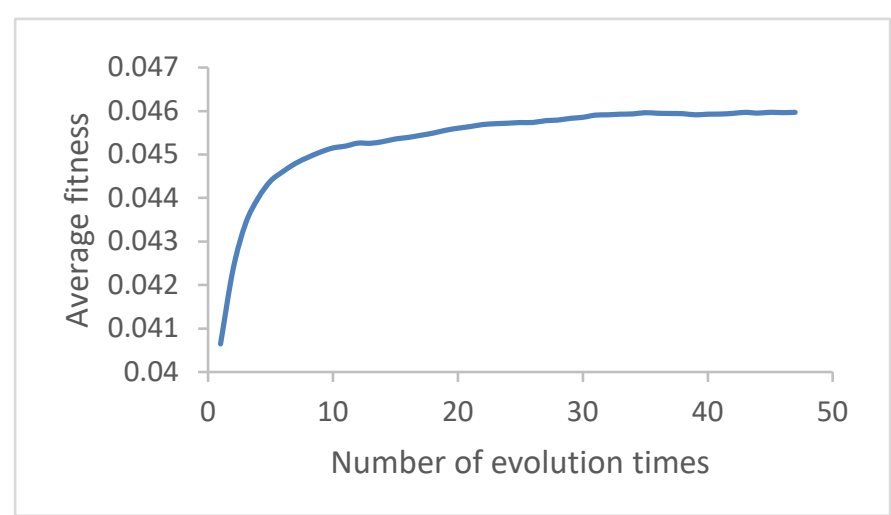

Figure 6. Evolution of GA population

The optimal results obtained for the parameters considered are:

- Corrective maintenance is necessary for maintaining an efficient system.

- Of the layout configurations considered, Figure 1 , having the maintenance site within the base is optimal due to the savings on land cost.

- Periodic maintenance should occur regularly at every 4 months.

- The total cost per year would be $£ 185729$ and the number of missions completed per year 8032 .

\section{CONCLUSIONS}

It has been demonstrated that combining CPN models and GA's is an effective and efficient approach to assessing the performance of a multi-AGV system. Using this approach the optimal combination of corrective and predictive maintenance can be determined. Also various possible configurations for the maintenance site can be considered. The work presented here will be extended to consider more complex AGV systems.

\section{REFERENCES}

1. P.R.Gutta, V.S.Chinthala, R.V.Manchoju, M.V.N. Charan, R. Purohit, "A review on facility layout design of an automated guided vehicle in flexible manufacturing system”, Materials Today :Proceedings, 5, 2018, pp39813986.

2. R. J. Gaskins and J. M. A. Tanchoco, "Flow path design for automated guided vehicle systems," Int. J Prod. Res., vol. 25, no. 5, pp. 667-676, 1987.

3. B. Beamon, "Performance, reliability, and performability of material handling systems," Int. J Prod. Res., vol. 36, no. 2, p. 377-393, 1998.

4. M. Tavana, H. Fazlollahtabar and R. Hassanzadeh, “A biobjective stochastic programming model for optimising automated material handling systems with reliability considerations," Int. J Prod. Res., vol. 52, no. 19, pp. 5597-5610, 2014.

5. M. Ebben, "Logistic control in automated transportation networks," University of Twente, Enschede, 2001.

6. R. Yan, L. M. Jackson and S. J. Dunnett, "Automated 
guided vehicle mission reliability modelling using a combined fault tree and Petri net approach", Int. $J A d v$. Man. Tech., 2017.

7. R. Yan, S. J. Dunnett and L. M. Jackson, "Novel methodology for optimising the design, operation and maintenance of a mult-agv system", Reliab. Eng. syst. Safety, vol. 178, pp130-139, 2018.

8. T. Nishi, R. Maeno, "Petri net decomposition approach to optimization of route planning problems for $\mathrm{AGV}$ systems", IEEE Transactions on Automation Science and Engineering 7(3): 2010, pp523-537

9. B. Reddy, C. Rao. "A hybrid multi-objective GA for simultaneous scheduling of machines and AGVs in FMS”, Int. J Adv. Man. Tech., vol 31, 602-613, 2006.

10. J.H. Holland, Adaptation in natural and artificial system: an introduction with application to biology, control and artificial intelligence: The University of Michigan Press, 1975.

\section{BIOGRAPHIES}

Rundong Yan MEng

Department of Aeronautical and Automotive Engineering Loughborough University

Loughborough, Leicestershire LE11 3TU UK

e-mail: R.Yan@lboro.ac.uk

Derek is a $\mathrm{PhD}$ researcher at Loughborough University. He obtained his Masters of Engineering (MEng) from Imperial College, London University, UK. The main focus of his current research is concerned with analysing the reliability of automated guided vehicles. Different analytical and theoretical techniques, including FMECA, Fault Tree Analysis, and Petri net model simulation, are conducted to identifying the key components and the work stages which are more vulnerable to failures. Of current interest is developing a simulation model for multi-AGV systems to understand and investigate the operation and interaction between AGVs.

\section{Sarah Dunnett, $\mathrm{PhD}$}

Department of Aeronautical and Automotive Engineering Loughborough University Loughborough, Leicestershire LE11 3TU UK

e-mail: s.j.dunnett@lboro.ac.uk

Sarah is a senior lecturer at Loughborough University and a member of the Control and Reliability Research Group. Her research has mainly focussed on system reliability assessment involving the application of a variety of techniques. Her work has also extended to the related areas of fault diagnostics and prognostics, automated reliability model generation, aerosol modelling, demand modelling and resource allocation.

Lisa Jackson, $\mathrm{PhD}$

Department of Aeronautical and Automotive Engineering

Loughborough University

Loughborough, Leicestershire LE11 3TU UK

e-mail: 1.m.jackson@lboro.ac.uk

Lisa is a Professor in the Dept. of Aeronautical \& Automotive Engineering at Loughborough University. She is a member of the Control and Reliability research group. Her research has focussed on multi-objective optimisation applied to safety system and process design, fault diagnostic and prognostic methods, systems reliability assessment; demand modelling and resource allocation. 